\title{
Optimal protocols and universal time-energy bound in Brownian thermodynamics
}

\author{
Yoseline Rosales-Cabara, ${ }^{1}$ Giovanni Manfredi, ${ }^{2, *}$ Gabriel Schnoering,,${ }^{1, \dagger}$ Paul-Antoine Hervieux, ${ }^{2, \sharp}$ \\ Laurent Mertz, ${ }^{3, \S}$ and Cyriaque Genet $\odot^{1, \pi}$ \\ ${ }^{1}$ Université de Strasbourg, CNRS, Institut de Science et d'Ingénierie Supramoléculaires, UMR 7006, F-67000 Strasbourg, France \\ ${ }^{2}$ Université de Strasbourg, CNRS, Institut de Physique et Chimie des Matériaux de Strasbourg, UMR 7504, F-67000 Strasbourg, France \\ ${ }^{3}$ NYU-ECNU Institute of Mathematical Sciences at NYU Shanghai, Shanghai 200062, China
}

(Received 9 June 2019; published 14 January 2020)

\begin{abstract}
We propose an optimization strategy to control the dynamics of a stochastic system transferred from one thermal equilibrium to another and apply it experimentally to a Brownian particle in an optical trap under compression. Based on a variational principle that treats the transfer duration and the expended work on an equal footing, our strategy leads to a family of protocols that is either optimally cheap for a given duration or optimally fast for a given energetic cost. This approach unveils a universal relation $\Delta t \Delta W \geqslant(\Delta t \Delta W)_{\mathrm{opt}}$ between the transfer duration and the expended work. We verify experimentally that the lower bound is reached only with the optimized protocols.
\end{abstract}

DOI: 10.1103/PhysRevResearch.2.012012

Controlling the transformation of equilibrium states is a major concern in stochastic energetics. Though still in its infancy, this is an important research area with promising applications both from classical and quantum perspectives [1-5]. Recent work demonstrated the possibility to control the evolution of a small system, forcing, for instance, a nano- or microsystem to evolve from one equilibrium state to another much faster than the relaxation time expected from the energy difference between the two equilibria [6-8]. From a mathematical viewpoint, this is an interesting optimal control problem, which can be studied using the Pontryagin's principle $[9,10]$.

Accelerated equilibration protocols have direct thermodynamic consequences. A protocol that reduces the transfer duration is necessarily more expensive energetically, so that the requirements of being fast and cheap cannot be satisfied simultaneously [6]. Earlier proposals have discussed the possibility to minimize the work expended through a transfer whose duration is initially fixed [1]. But with duration and work not treated on an equal footing, these proposals prevent one from deriving and exploiting the mutually exclusive relation between a protocol's duration and its energetic cost. Such a relation is, however, of paramount importance to design

\footnotetext{
*giovanni.manfredi@ipcms.unistra.fr

†Present address: Laboratory of Thermodynamics in Emerging Technologies, Department of Mechanical and Process Engineering, ETH Zürich, Sonneggstrasse 3, CH-8092 Zürich, Switzerland.

"hervieux@ipcms.unistra.fr

\$laurent.mertz@nyu.edu

Igenet@unistra.fr
}

Published by the American Physical Society under the terms of the Creative Commons Attribution 4.0 International license. Further distribution of this work must maintain attribution to the author(s) and the published article's title, journal citation, and DOI. protocols that are optimized from both points of view. The possibility for optimal control turns out to be particularly relevant in the field of stochastic engines, where it is necessarily related to the global figure of merit of the system $[11,12]$.

In order to derive such optimal protocols, we adopt an approach that treats both the duration of the transfer and the expended work in a completely symmetric way. Our strategy is implemented on an optical trap that determines a simple framework (harmonic potentials) where theory can be elaborated in perfect tandem with experiments. This provides a capacity for exploring optimization with two ingredients. First, each protocol is defined by a path in the phase space $(\kappa, s)$, where $\kappa$ is the stiffness of the optical trap and $s$ is the variance of the position of the particle. Second, we construct a functional $J[\kappa, s]$ that is composed of two terms, corresponding, respectively, to the total work and total duration, with a Lagrange multiplier $\lambda$ regulating the trade-off between these two quantities. Minimizing the above functional with fixed boundary conditions yields the desired optimal protocol $\kappa_{\lambda}(s)$. For instance, $\lambda \gg 1$ gives a protocol of low energetic cost but long duration, while $\lambda \ll 1$ gives a fast protocol requiring a large amount of work.

Remarkably, this treatment leads to a universal relation $\Delta t \Delta W \geqslant(\Delta t \Delta W)_{\text {opt }}$ between the transfer duration $\Delta t$ and the expended work $\Delta W$ (in excess of the equilibrium freeenergy difference), where the lower bound depends exclusively on the initial and final states and is reached only under optimal control conditions. This result unveils a fundamental feature that underpins all optimization procedures in stochastic thermodynamics $[13,14]$. In particular, we demonstrate that protocols optimized under the further constraint of a limited speed of variation for $\kappa(s)$ do converge towards work-duration optimal ones.

We optically trap a Brownian particle - a polystyrene microsphere of radius $500 \mathrm{~nm}$-in water at room temperature inside a harmonic potential; see the Supplemental Material, Sec. A [15], and [16,17]. We record the instantaneous motion 
$x(t)$ of the microsphere along the trap optical axis. With the trap stiffness $\kappa$ proportional to the trapping laser intensity $I$, it is possible to define, by modulating $I(t)$, a given protocol $\kappa(t)$ for the transfer from an initial thermal equilibrium at time $t_{i}$ to a final equilibrium at time $t_{f}$. By performing a series of $N$ identical protocols on the trapped microsphere, we build a statistical ensemble of trajectories that yields a probability density function (PDF) of positions $x$. The dynamics of the system is described by the variance $s(t)$ extracted from the PDF of positions, which evolves as $[18,19]$

$$
\gamma \frac{d s}{d t}=-2 \kappa(t) s+2 D \gamma,
$$

where $\gamma$ is the Stokes drag coefficient and $D=k_{B} T / \gamma \sim$ $0.4 \mu \mathrm{m}^{2} / \mathrm{s}$ is the Brownian diffusion coefficient fixed by the temperature $T$ (room temperature) of water and the Boltzmann constant $k_{B}$.

Equation (1) fully determines the statistical properties of the system where the initial and final equilibria correspond to the stationary solutions $s_{i} \kappa_{i}=s_{f} \kappa_{f}=k_{B} T$ with the PDF that remain Gaussian for all intermediate times between $t_{i}$ and $t_{f}$. The cumulative energetics involved during the protocol is directly related to the time evolution of $s(t)$, giving the ensemble average expended work $W(t)=\frac{1}{2} \int_{t_{i}}^{t} d t s(t) \dot{\kappa}(t)$ and dissipated heat $Q(t)=-\frac{1}{2} \int_{t_{i}}^{t} d t \dot{s}(t) \kappa(t)[14,20]$.

Our purpose is to control the dynamics of the microsphere so that the transfer between the two equilibria is optimal with respect to both duration and energetics. If one instantaneously switches the trap stiffness from $\kappa_{i}$ to $\kappa_{f}>\kappa_{i}$ (i.e., closing the trap in a steplike way), the typical relaxation time to the new equilibrium is given by $\tau_{\text {relax }}=2 \gamma / \kappa_{f}$. Our optimization strategy starts with the idea of using the variance $s$ as the independent variable of the problem, instead of the time $t$. This is possible whenever the function $s(t)$ is monotonic and it enables us to express the control parameter as $\kappa(t)=\hat{\kappa}[s(t)]$. The advantage of this approach is that we can easily write down, as functionals of $\hat{\kappa}(s)$, both the transfer duration,

$$
\Delta t[\hat{\kappa}(s)] \equiv t_{f}-t_{i}=\frac{1}{2} \int_{s_{i}}^{s_{f}} \frac{\gamma d s}{D \gamma-s \hat{\kappa}(s)},
$$

and the expended work,

$$
W[\hat{\kappa}(s)]=-\frac{1}{2} \int_{s_{i}}^{s_{f}} \hat{\kappa}(s) d s+\frac{1}{2}\left(\kappa_{f} s_{f}-\kappa_{i} s_{i}\right),
$$

where the second term on the right-hand side vanishes because the initial and final configurations are thermally equilibrated.

Next, we define the functional to be minimized as twice the sum of $W$ and $\Delta t$,

$$
J[\hat{\kappa}(s)]=\int_{s_{i}}^{s_{f}} \frac{\gamma d s}{D \gamma-s \hat{\kappa}(s)}-\lambda \int_{s_{i}}^{s_{f}} \hat{\kappa}(s) d s,
$$

where $\lambda$ is a Lagrange multiplier that regulates the trade-off between transfer duration and work. Within this framework, the optimization strategy can be interpreted as the search for the trajectory in the $(s, \kappa)$ space that minimizes $J[\hat{\kappa}(s)]$ while keeping the extrema fixed at equilibrium, i.e., $s_{i} \kappa_{i}=$ $s_{f} \kappa_{f}=k_{B} T=D \gamma$. Once written as $J=\int_{s_{i}}^{s_{f}} L[s, \hat{\kappa}(s)] d s$, this functional can be minimized using the standard Euler-Lagrange equation $d / d s\left(\partial L / \partial \hat{\kappa}^{\prime}\right)-\partial L / \partial \hat{\kappa}=0$, where $\hat{\kappa}^{\prime} \equiv d \hat{\kappa} / d s$, yielding the following solution:

$$
s \hat{\kappa}(s)=D \gamma+\sqrt{\gamma s / \lambda},
$$

for a protocol that eventually closes the trap with $\kappa_{f}>\kappa_{i}$ [21].

Equation (5) encapsulates the main result obtained so far. For instance, the quasistatic solution $(\dot{s} \approx 0)$ is obtained by taking $\lambda \rightarrow \infty$, which yields an infinite duration but the smallest possible expended work, $W_{\mathrm{QS}}=\frac{1}{2} D \gamma \ln \left(\kappa_{f} / \kappa_{i}\right)$, equal to the free-energy difference, as expected for a quasistatic process. For finite $\lambda$ and making use of Eq. (5), Eq. (1) can be rewritten as $\dot{s}=-2 \sqrt{s / \gamma \lambda}$, which possesses the general solution $s(t)=\left(\sqrt{s_{i}}-t / \sqrt{\gamma \lambda}\right)^{2}$. Inserting this expression into Eq. (5) yields the optimal evolution of the trap stiffness,

$$
\kappa(t)=\frac{D \gamma+\sqrt{\gamma s_{i} / \lambda}-t / \lambda}{\left(\sqrt{s_{i}}-t / \sqrt{\gamma \lambda}\right)^{2}},
$$

which defines our protocol for the optimized transfer.

It is important to stress that in our case, the Euler-Lagrange equation is purely algebraic so that one cannot enforce the initial and final conditions. Thus, except for the quasistatic limit, Eq. (6) does not satisfy the conditions for which the system is at thermal equilibrium in the initial and final states. In order to ensure that $s_{i, f} \kappa_{i, f}=D \gamma$, we need to add to Eq. (6) two discontinuities (as already noticed in $[1,22]$ ). The optimal protocol thus consists of three successive sequences:

(1) At time $t=t_{i}$, the trap stiffness is suddenly changed from $\kappa_{i}=D \gamma / s_{i}$ (initial equilibrium) to $\kappa\left(t_{i}^{+}\right)=\kappa_{i}^{+}$, such that $\kappa_{i}^{+}-\kappa_{i}=\sqrt{\gamma /\left(\lambda s_{i}\right)}$, while keeping the variance equal to $s_{i}$.

(2) Between $t_{i}^{+}$and $t_{f}^{-}$, the stiffness varies according to Eq. (6), reaching $\kappa\left(t_{f}^{-}\right) \equiv \kappa_{f}^{-}=D \gamma / s_{f}+\sqrt{\gamma /\left(\lambda s_{f}\right)}$.

(3) At time $t=t_{f}$, the stiffness is suddenly changed from $\kappa_{f}^{-}$to $\kappa_{f}=D \gamma / s_{f}$ (final equilibrium), while keeping the variance equal to $s_{f}$.

Experimentally, we have performed, on the trapped microsphere, a series of $N=2 \times 10^{4}$ such optimal protocols, forcing the microsphere to relax to thermal equilibrium within a time $\Delta t$ chosen to be shorter than $\tau_{\text {relax }}$. Figure 1 displays $s(t)$ between two equilibria for a shortened duration $\Delta t \sim$ $\tau_{\text {relax }} / 10$, where $\tau_{\text {relax }}=2 \gamma / \kappa_{f}=3.22 \pm 0.09 \mathrm{~ms}$. The reduction in $s(t)$ from its initial value corresponds to the fact that the trap is stiffer at the end of the protocol with $\kappa_{f} / \kappa_{i} \sim 1.85$. It is in excellent agreement with the theory.

This reduction in the transfer duration has an energetic cost that can be evaluated for each sequence and measured experimentally by evaluating the cumulative work $W(t)$ from the recorded evolution of $\hat{\kappa}(s)$. Figure 2 shows the time evolution of $W(t)$ split into three sequences. First, the quantity of work, $W^{(1)}=s_{i}\left(\kappa_{i}^{+}-\kappa_{i}\right) / 2$, is injected instantaneously into the system at the time $t_{i}$ as the trap is suddenly stiffened from $\kappa_{i}$ to $\kappa_{i}^{+}$. During the second sequence, the injection of work continues as the trapping volume is progressively reduced, reaching $W^{(2)}=W_{\mathrm{QS}}+\sqrt{\gamma / \lambda}\left[\left(s_{i}^{3 / 2}-s_{f}^{3 / 2}\right) / 3-\right.$ $\left.\left(s_{i}^{1 / 2}-s_{f}^{1 / 2}\right) / 2\right]$ at $t=t_{f}^{-}$, when $\kappa=\kappa_{f}^{-}$. Finally, the trap is suddenly expanded at $t=t_{f}$, and the system instantly reaches its final equilibrium state, delivering to the thermal bath a quantity of work equal to $W^{(3)}=s_{f}\left(\kappa_{f}-\kappa_{f}^{-}\right) / 2$. For $t>t_{f}$, the thermal steady state is characterized by $W=Q$. 


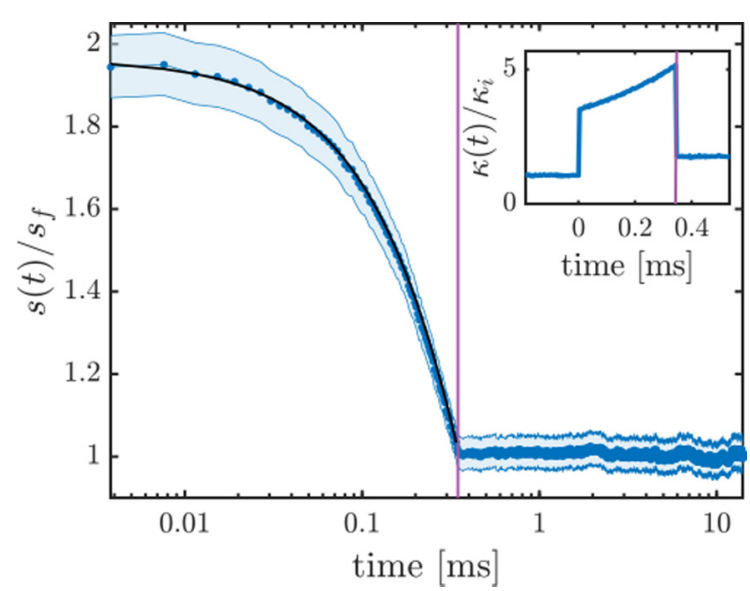

FIG. 1. Time evolution of the variance $s(t)$ for an optimal protocol of duration of $\Delta t=3.47 \times 10^{-4} \mathrm{~s} \sim \tau_{\text {relax }} / 10$, indicated by the vertical solid line. Starting at time $t_{i}$ at thermal equilibrium with $\kappa_{i}=2.77 \pm 0.08 \mathrm{pN} / \mu \mathrm{m}$ and a mean $s_{i}=1.48 \times 10^{-15} \mathrm{~m}^{2}$, the variance is extracted from the PDF of positions and normalized to the final equilibrium state associated with the plateau-averaged value $s_{f}=7.75 \times 10^{-16} \mathrm{~m}^{2}$ reached after $t_{f}$, corresponding to $\kappa_{f}=$ $5.22 \pm 0.15 \mathrm{pN} / \mu \mathrm{m}$. The Lagrange multiplier $\lambda=(1.27 \pm 0.02) \times$ $10^{17} \mathrm{~s} / \mathrm{J}$ associated with this protocol is determined by the set of values $\left(\Delta t, s_{i}, s_{f}\right)$ and Eq. (8). The superimposed black continuous line is the theoretical time evolution of the optimized variance, $s(t)=\left(\sqrt{s_{i}}-t / \sqrt{\gamma \lambda}\right)^{2}$, calculated with the measured values. The experimental error bars correspond to a $95 \%$ confidence interval for $s(t)$, including calibration uncertainties (see more details in the Supplemental Material, Sec. A [15]). Inset: The experimental optimal protocol $\kappa(t)$, normalized to the initial stiffness $\kappa_{i}$.

In contrast, heat is continuously dissipated from the microsphere to the thermal bath, as seen in Fig. 2, from the monotonic increase of the ensemble average cumulative heat $Q(t)$ throughout the protocol. This evolution is almost exactly linear in time, which corresponds to a constant production of entropy. We stress again that the experimentally measured values of the heat and work injected in and extracted from the system are in excellent agreement with the theoretical predictions.

The total work expended throughout the optimal protocol is evaluated by adding the contributions from each of the three sequences described above. One obtains

$$
W_{\mathrm{opt}}=W_{\mathrm{QS}}+\sqrt{\gamma / \lambda}\left(s_{i}^{1 / 2}-s_{f}^{1 / 2}\right) .
$$

Similarly, the total duration of the optimal protocol is obtained by inserting Eq. (5) into Eq. (2), yielding

$$
\Delta t_{\mathrm{opt}}=\sqrt{\gamma \lambda}\left(s_{i}^{1 / 2}-s_{f}^{1 / 2}\right) .
$$

The above expressions clearly show that our optimization procedure is perfectly symmetric as far as duration and work are concerned and that the trade-off between these two quantities is governed by the Lagrange multiplier $\lambda$. Indeed, one can choose $\lambda$ using Eq. (8) to fix the total duration and then the minimum expended work will be given by Eq. (7); or, alternatively, one can determine $\lambda$ through Eq. (7) to fix the total work and then the minimum duration of the process will be given by Eq. (8). This leads us to define the "excess

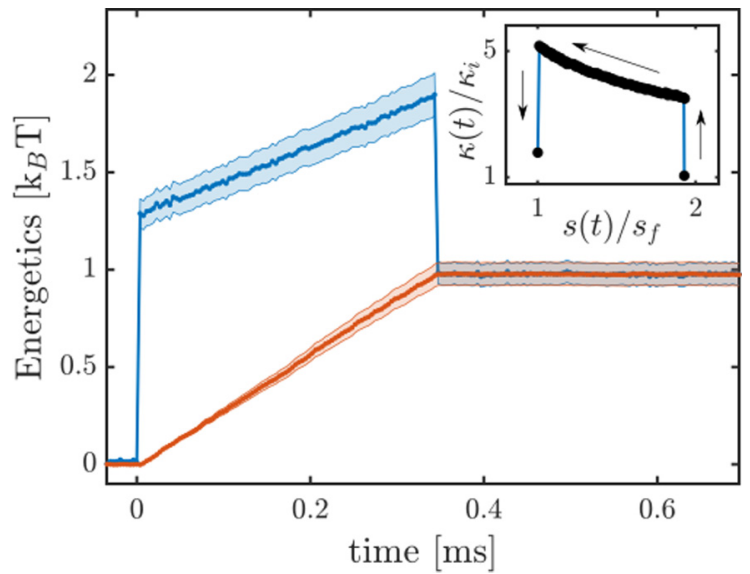

FIG. 2. Energetics associated with the optimal protocol described in Fig. 1 for $\Delta t \sim \tau_{\text {relax }} / 10$. The ensemble average cumulative work $W(t)$ (blue curve) and heat $Q(t)$ (red curve) are measured with respect to the initial thermal equilibrium. The experimental error bars are determined by the propagation of variance and calibration (i.e., stiffness) uncertainties (see the Supplemental Material, Sec. A [15]). At $t>t_{f}$, the system has reached a thermal steady state with $W\left(t>t_{f}\right)=(0.981 \pm 0.059) k_{B} T \sim Q\left(t>t_{f}\right)=$ $(0.983 \pm 0.060) k_{B} T$. Inset: The control parameter $\hat{\kappa}(s)$, with the arrows corresponding to the time evolution.

work" of the optimal protocol as $\Delta W_{\mathrm{opt}} \equiv W_{\mathrm{opt}}-W_{\mathrm{QS}}$ and to note that the product $\Delta t_{\mathrm{opt}} \Delta W_{\mathrm{opt}}=\gamma / 2\left(s_{i}^{1 / 2}-s_{f}^{1 / 2}\right)^{2}$ is independent of $\lambda$ and only depends on the initial and final states [23]. This equality fixes the mutually exclusive relation between transfer duration and expended work under optimal control. It corresponds to the frontier value of a universal exclusion region, $\Delta t \Delta W \geqslant \gamma / 2\left(\sqrt{s_{i}}-\sqrt{s_{f}}\right)^{2}$, that bounds from below all protocols that are not optimal.

The frontier can be drawn experimentally by measuring $\Delta W_{\text {opt }}$ for a series of optimal protocols with different transfer durations $\Delta t$. By normalizing each measured value of $\Delta W_{\mathrm{opt}}$ to the associated value of $\gamma / 2\left(\sqrt{s_{i}}-\sqrt{s_{f}}\right)^{2}$, one can test the universal nature of the bound. This is clearly confirmed in Fig. 3, with all the optimal solutions implemented experimentally falling precisely on the $1 / \Delta t$ curve. To further prove that the frontier corresponds to a lower bound, we have verified experimentally that the $\{\Delta t, \Delta W\}$ coordinates of typical nonoptimal protocols all fall above the expected bound, as displayed in Fig. 3.

A salient feature of our optimal control procedure is represented by the sudden jumps in stiffness that have to augment the solution of Eq. (6) in order to comply with thermally equilibrated initial and final configurations. From an experimental point of view, such discontinuities do not constitute a weakness of the procedure, as they correspond to finite and measurable quantities of work exchanged between the bath and the system $[10,24]$. But it is interesting to stress that one asset of our variational strategy is its capacity to construct smooth protocols that are as close as desired to the optimal ones. For this, we need to control the derivatives of the function $\hat{\kappa}(s)$ by adding the gradient term $\int_{s_{i}}^{s_{f}}\left|\hat{\kappa}^{\prime}(s)\right|^{2} d s$ to the functional $J[\hat{\kappa}(s)]$ in Eq. (4), with a second Lagrange multiplier $\varepsilon$ that acts as a regularization parameter by removing 


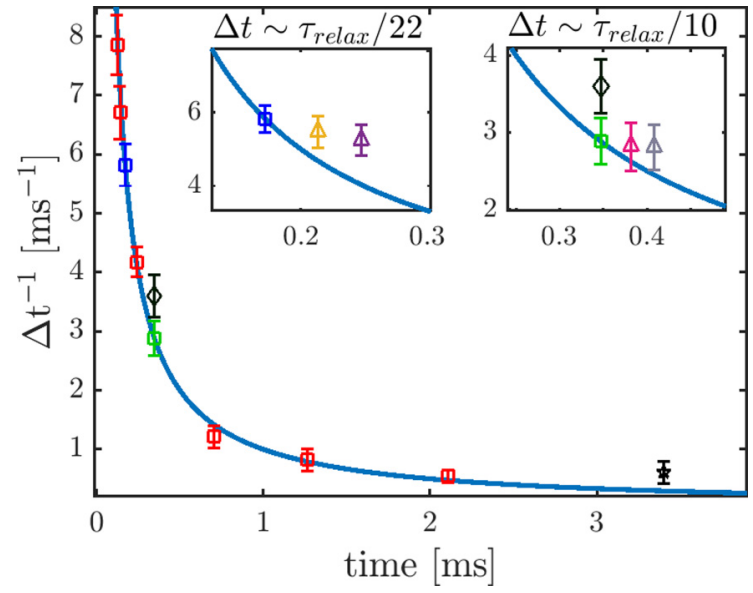

FIG. 3. Extracted excess works $\Delta W_{\text {opt }}$ revealing the mutually exclusive relation between $\Delta W_{\mathrm{opt}}$ and $\Delta t$ for a series of protocols. Optimal protocols (squares) for transfer durations $\Delta t=\tau_{\text {relax }} / n$, with successively $n \sim 34,30,22,16,10,6,3$, and 2 , "engineered swift equilibration" protocol [6] (black diamond) defined for $\Delta t=$ $3.47 \times 10^{-4} \mathrm{~s} \sim \tau_{\text {relax }} / 10$ (also in inset), steplike protocol (black star) at $\tau_{\text {relax }} \sim 3.22$; see details in the Supplemental Material, Sec. B [15]. All optimized coordinates $\left\{\Delta t, \Delta W_{\text {opt }} /\left[\gamma / 2\left(\sqrt{s_{i}}-\right.\right.\right.$ $\left.\left.\sqrt{s_{f}}\right)^{2}\right]$ \} precisely fall (within error bars) on the $1 / \Delta t$ curve, whereas the values corresponding to the nonoptimal protocol fall above the universal bound. Insets: coordinates measured for smooth (thus suboptimal) protocols for $n \sim 22$ and $n \sim 10$, and smoothness parameters $\varepsilon=5 \times 10^{-6}, \varepsilon=10^{-6}$, and $\varepsilon=0$, expressed in units of $s_{i}^{2} /\left(D \kappa_{i}^{2}\right)$. Such smooth protocols are defined using the same Lagrange multiplier $\lambda$ as their associated optimal protocols. For each case, the product $\Delta t \Delta W$ converges to the optimal lower bound (solid blue line) as $\varepsilon \rightarrow 0$.

the discontinuities in $\hat{\kappa}(s)$. Hence, we arrive at the modified Euler-Lagrange equation $2 \varepsilon d^{2} \hat{\kappa} / d s^{2}=\gamma s /(D \gamma-s \hat{\kappa})^{2}-\lambda$, which can be solved numerically as a boundary value problem, with initial and final conditions at thermal equilibrium, $\hat{\kappa}\left(s_{i, f}\right)=D \gamma / s_{i, f}$. Once the solution $\hat{\kappa}(s)$ is known, the time evolution of the variance $s(t)$ is found by integrating Eq. (1) (more details are given in the Supplemental Material, Sec. C [15]).

Using the same values of $\lambda$ that defined the optimal protocols with, respectively, $\Delta t \sim \tau_{\text {relax }} / 22$ and $\Delta t \sim \tau_{\text {relax }} / 10$ (Fig. 3, inset), we implemented two smooth protocols for two different values of the Lagrange multiplier. As shown in Fig. 4, the smooth protocols closely follow the optimal ones, except near the beginning and the end of the process, where they approach the equilibrium states in a continuous way. For the same value of $\lambda$, smooth protocols give slightly longer transfer durations than the optimal protocol but, as expected, the expended work is slightly smaller in the smooth case than in the optimized limit. The nonoptimal character of the smooth protocols is clearly seen in the insets of Fig. 3, where all $\{\Delta t, \Delta W\}$ coordinates lie above the universal bound, and only converge towards it in the $\varepsilon \rightarrow 0$ limit.

In conclusion, we have devised a family of optimal protocols that transfer an optically trapped microsphere between

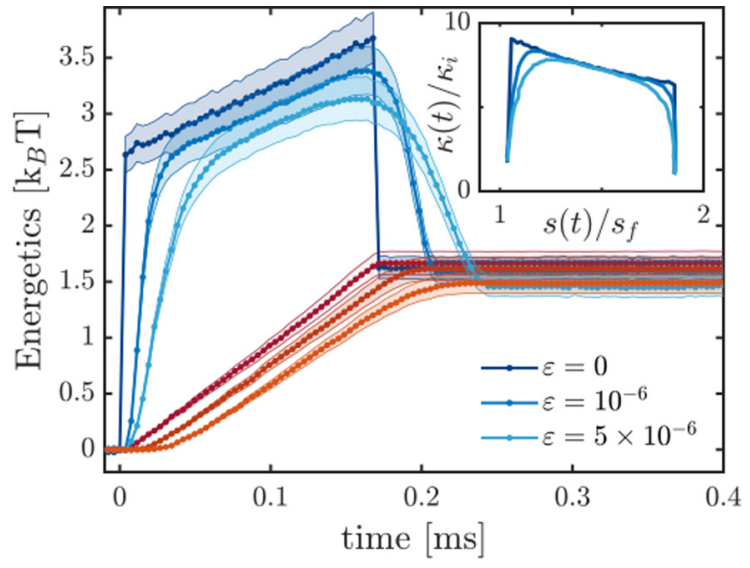

FIG. 4. Comparison of the cumulative energetics-expended work $W(t)$ (blue curves) and dissipated heat $Q(t)$ (red curves) between an optimal protocol defined for $\Delta t=1.72 \times 10^{-4} \mathrm{~s} \sim$ $\tau_{\text {relax }} / 22$ and two smooth protocols with $\varepsilon=5 \times 10^{-6}$ and $\varepsilon=10^{-6}$, expressed in units of $s_{i}^{2} /\left(D \kappa_{i}^{2}\right)$, and identical value of $\lambda=(2.97 \pm$ $0.12) \times 10^{16} \mathrm{~s} / \mathrm{J}$. As seen in the insets of Fig. 3, although the smooth protocols involve slightly less expended work $\left(1.36 \pm 0.06 k_{B} T\right.$ for $\varepsilon=5 \times 10^{-6}$ and $1.65 \pm 0.06 k_{B} T$ for $\left.\varepsilon=10^{-6}\right)$ than the optimal one $\left(1.69 \pm 0.06 k_{B} T\right)$, they correspond to longer transfer durations $\left(2.48 \times 10^{-4} \mathrm{~s}\right.$ for $\varepsilon=5 \times 10^{-6}$ and $2.14 \times 10^{-4} \mathrm{~s}$ for $\left.\varepsilon=10^{-6}\right)$. Inset: Superimposed evolutions $\kappa(t)$ vs $s(t)$ for the three protocols, showing the continuous nature of the smooth protocol and illustrating the progressive convergence to the optimal protocol in the $\varepsilon \rightarrow 0$ limit. For each protocol, the curves are normalized to the corresponding $\kappa_{i}$ for $\kappa(t)$ and $s_{f}$ for $s(t)$.

two equilibria, minimizing both the transfer duration and the associated energetic cost. Within such protocols, the tradeoff between duration and work can be modulated at will by tuning a single Lagrange multiplier given by our variational approach. A key result of our work is to show that the product $\Delta t \Delta W$ is bounded from below, in a way reminiscent of energy-time uncertainty relations. Similar bounds were noticed in earlier works $[13,14]$, but only for some special cases. Here, our bound is universal (it depends exclusively on the initial and final states) and is only reached for the optimal protocol. Further extending the present results to quantum systems may open new interesting perspectives in the burgeoning field of quantum stochastic thermodynamics [25-28].

This work was supported in part by Agence Nationale de la Recherche (ANR), France, ANR Equipex Union (Grant No. ANR-10-EQPX-52-01), the Labex NIE projects (Grant No. ANR-11-LABX-0058-NIE), and USIAS within the Investissements d'Avenir program (Grant No. ANR-10-IDEX0002-02). Y.R.-C. is a member of the International Doctoral Program of the Initiative d'Excellence of the University of Strasbourg, whose support is acknowledged. L.M. is supported by the National Natural Science Foundation of China, Research Fund for International Young Scientists under the Project No. 1161101053 and the Young Scientist Program under the Project No. 11601335. 
[1] T. Schmiedl and U. Seifert, Optimal Finite-Time Processes in Stochastic Thermodynamics, Phys. Rev. Lett. 98, 108301 (2007).

[2] Xi Chen, A. Ruschhaupt, S. Schmidt, A. del Campo, D. GuéryOdelin, and J. G. Muga, Fast Optimal Frictionless Atom Cooling in Harmonic Traps: Shortcut to Adiabaticity, Phys. Rev. Lett. 104, 063002 (2010).

[3] E. Aurell, K. Gawedzki, C. Mejía-Monasterio, R. Mohayaee, and P. Muratore-Ginanneschi, Refined second law of thermodynamics for fast random processes, J. Stat. Phys. 147, 487 (2012).

[4] U. Seifert, Stochastic thermodynamics, fluctuation theorems and molecular machines, Rep. Prog. Phys. 75, 126001 (2012).

[5] S. J. Weber, A. Chantasri, J. Dressel, A. N. Jordan, K. W. Murch, and I. Siddiqi, Mapping the optimal route between two quantum states, Nature (London) 511, 570 (2014).

[6] I. A. Martínez, A. Petrosyan, D. Guéry-Odelin, E. Trizac, and S. Ciliberto, Engineered swift equilibration of a Brownian particle, Nat. Phys. 12, 843 (2016).

[7] M. Chupeau, B. Besga, D. Guéry-Odelin, E. Trizac, A. Petrosyan, and S. Ciliberto, Thermal bath engineering for swift equilibration, Phys. Rev. E 98, 010104(R) (2018).

[8] A. Le Cunuder, I. A. Martínez, A. Petrosyan, D. Guéry-Odelin, E. Trizac, and S. Ciliberto, Fast equilibrium switch of a micro mechanical oscillator, Appl. Phys. Lett. 109, 113502 (2016).

[9] L. S. Pontryagin, V. G. Boltyanskii, R. V. Gamkrelidze, and E. F. Mishchenko, The Mathematical Theory of Optimal Processes, translated by K. N. Trirogof, edited by L. W. Neustadt (Interscience/Wiley, New York, 1962), pp. viii+360.

[10] C. A. Plata, D. Guéry-Odelin, E. Trizac, and A. Prados, Optimal work in a harmonic trap with bounded stiffness, Phys. Rev. E 99, 012140 (2019).

[11] V. Blickle and C. Bechinger, Realization of a micrometer-sized stochastic heat engine, Nat. Phys. 8, 143 (2011).

[12] I. A. Martínez, E. Roldán, L. Dinis, D. Petrov, J. M. R. Parrondo, and R. A. Rica, Brownian carnot engine, Nat. Phys. 12, 67 (2015).

[13] K. Sekimoto, Stochastic Energetics (Springer-Verlag, Berlin, 2010).

[14] S. Ciliberto, Experiments in Stochastic Thermodynamics: Short History and Perspectives, Phys. Rev. X 7, 021051 (2017).

[15] See Supplemental Material at http://link.aps.org/supplemental/ 10.1103/PhysRevResearch.2.012012 for a description of the optical trap used in this work, its calibration and associated uncertainties, including a discussion on Eq. (1) in the main text governing the variance $s(t)$, statistical and energetics uncertainties (Sec. A), a comparison between optimal, steplike and "engineered swift equilibration" [6] protocols (Sec. B), and a detailed discussion on smooth (suboptimal) protocols (Sec. C).

[16] G. Schnoering, L. V. Poulikakos, Y. Rosales-Cabara, A. Canaguier-Durand, D. J. Norris, and C. Genet, ThreeDimensional Enantiomeric Recognition of Optically Trapped Single Chiral Nanoparticles, Phys. Rev. Lett. 121, 023902 (2018).

[17] G. Schnoering, Y. Rosales-Cabara, H. Wendehenne, A. Canaguier-Durand, and C. Genet, Thermally Limited Force Microscopy on Optically Trapped Single Metallic Nanoparticles, Phys. Rev. Appl. 11, 034023 (2019).

[18] A. Manoliu and C. Kittel, Correlation in the Langevin theory of Brownian motion, Am. J. Phys. 47, 678 (1979).

[19] C. Gardiner, Stochastic Methods (Springer-Verlag, Berlin, 2009).

[20] K. Sekimoto, Langevin equation and thermodynamics, Prog. Theor. Phys. Suppl. 130, 17 (1998).

[21] In order to satisfy $\Delta t>0$, a negative sign should be used on the right-hand side of Eq. (5) when $s_{f}>s_{i}$ (opening trap, $\kappa_{f}<\kappa_{i}$ ), whereas a positive sign should be used when $s_{f}<s_{i}$ (closing trap, $\kappa_{f}>\kappa_{i}$ ).

[22] T. Schmiedl and U. Seifert, Efficiency at maximum power: An analytically solvable model for stochastic heat engines, Europhys. Lett. 81, 20003 (2008).

[23] This "excess work" corresponds to the concept of "dissipative work" proposed, for instance, in [29,30] and more commonly found. We, however, keep this expression because it illustrates our aim to optimally reduce the duration and the energetic cost of the protocol.

[24] E. Aurell, C. Mejía-Monasterio, and P. Muratore-Ginanneschi, Boundary layers in stochastic thermodynamics, Phys. Rev. E 85, 020103(R) (2012).

[25] C. Elouard, D. A. Herrera-Martí, A. Auffèves, and M. Clusel, The role of quantum measurement in stochastic thermodynamics, Nat. Quantum Inf. 3, 9 (2017).

[26] S. Vinjanampathy and J. Anders, Quantum thermodynamics, Contemp. Phys. 57, 545 (2016).

[27] J. Roßnagel, S. T. Dawkins, K. N. Tolazzi, O. Abah, E. Lutz, F. Schmidt-Kaler, and K. Singer, A single-atom heat engine, Science 352, 325 (2016).

[28] V. Cavina, A. Mari, A. Carlini, and V. Giovannetti, Optimal thermodynamic control in open quantum systems, Phys. Rev. A 98, 012139 (2018).

[29] C. Jarzynski, Nonequilibrium Equality for Free Energy Differences, Phys. Rev. Lett. 78, 2690 (1997).

[30] C. Jarzynski, Comparison of far-from-equilibrium work relations, C. R. Phys. 8, 495 (2007). 